histamine form (almost immediate and very steep contraction) obtained.

If the aqueous humour is not transferred to the gut immediately after collection, but is left at room temperature for 5-15 min., the onset of contraction is less delayed, and the contraction reaches its maximum in a shorter time.

If the aqueous humour is left for two to three hours at room temperature, it often causes an immediate onset of a rapid contraction, which is very similar to the histamine effect. The height of the contraction corresponded to amounts of histamine of the same order of magnitude as those found by Emmelin and Palm, and by Emmelin.

My first impression that this phenomenon was due to the liberation of histamine from an inactive complex found no justification. Addition of 'Neoantergan' to a final concentration of $1: 4 \times 10^{6}$ did not influence the contraction caused by aqueous humour $2.5 \mathrm{hr}$. old, whereas it completely abolished the effects of a corresponding amount of histamine.

This subject will be more fully described in Acta Ophthalmologica.

Ophthalmological Clinic, University of Lund. May 28.

'Emmelin, N., and Palm, E., Arta Ophtalm., 22, ii, 117 (1944). ${ }^{2}$ Fmmelin, N., Acta Physiol. Scand., 11, Supp. xxxiv (1945).

\title{
The Provitamin D in Clams
}

THE communication on the "Conversion of Cholesterol to Provitamin $\mathrm{D}_{3}$ in vivo" by Mary Scott, J. Glover and R. A. Morton in Nature of April 2, p. 530, prompts us to publish our results for the provitamin in clams. In order to obtain experimental evidence for the hypothesis that the provitamin $\mathrm{D}$ is essentially of endogenous origin, we investigated several years ago the sterols of the viscera and of the eviscerated body of clams. At the time when the clams were collected (late summer) their intestinal tract was filled with a dark green paste, indicating that the food consisted predominantly of chlorophyll-bearing micro-organisms such as algæ. The sterols isolated from the viscera contained 21.4 per cent provitamin D (determined spectroscopically and calculated as ergosterol). On the other hand, the sterols from the eviscerated body contained only 7.8 per cent provitamin D. Based on the weight of the fresh material, the viscera contained 0.0418 per cent provitamin $\mathrm{D}$ and the eviscerated body $0 \cdot 0163$ per cent. The total provitamin D content of the clams was found to be $0 \cdot 019$ per cent, and 24 per cent of the total provitamin $D$ was in the viscera.

In order to gain information as to the chemical nature of the provitamin D in clams, identical amounts of provitamin $D$ in the crude sterol fractions were irradiated with ultra-violet light, and the vitamin $D$ formed was dissolved in identical amounts of corn oil and tested for antirachitic activity both on rats (two experiments) and on chicks. The results obtained are summarized in the accompanying table :

Irradiated provitamin D from viscera

Irradiated provitamin $\mathrm{D}$

from eviscerated body

$\begin{array}{cc}\begin{array}{c}\text { 1. Rat test } \\ \text { U.S.P. }\end{array} & \begin{array}{c}\text { 2. Rat test } \\ \text { U.S.P. } \\ \text { units/gm. }\end{array} \\ \begin{array}{cc}\text { units/gm. } \\ 1,450\end{array} & 1,500 \\ 1,300 & 1,400\end{array}$

We have interpreted these findings as indicating that there is no difference in the type of provitamin D present in the viscera and the eviscerated body. We are also inclined to assume that they lend additional weight to the working hypothesis that the provitamin $\mathrm{D}$ is of endogenous origin. If a substantial part of the provitamin $D$ present in the viscera had been of exogenous origin, we would have expected to find a lower 'chick' efficiency for the vitamin D prepared from the provitamin from the viscera than for the vitamin $D$ prepared from the eviscerated body. Finally, we conclude that the provitamin D present in clams is neither pure ergosterol nor pure 7-dehydrocholesterol. If the provitamin were essentially ergosterol, we should have obtained a considerably lower activity for chicks than for rats, and if the provitamin were essentially 7-dehydrocholesterol we should have obtained a considerably higher 'chick' activity $^{1}$. Our results leave open the question whether the provitamin $\mathrm{D}$ in clams is a mixture of ergosterol with 7-dehydrocholesterol or a new provitamin $\mathrm{D}$, or a mixture of various known and unknown provitamins $D$.

We wish to acknowledge the assistance of W. F. Marlow, T. Parsons, jun., E. L. Rohdenburg, J. P. Marvel and G. H. Kennedy in this investigation.

HANS R. ROSENBERG

Biological Laboratory,

E. I. duPont de Nemours and Co.,

New Brunswick, N.J. April 22.

${ }^{1}$ Waddell, J., and Kennedy, G. H., J. A8soc. Official Agric. Chem., $30,190(1947)$.

\section{Shock and Mitotic Activity in Mice}

IT has been argued that the concentration of sugar (or glycogen) within a tissue is a critical factor in controlling the rate of mitosis ${ }^{1}$. An abundance of sugar induces active cell division, while a sugar deficiency results in mitosis depression. Such a depression follows treatment with insulin, which lowers the blood sugar level; phloridzin, which interferes with phosphorylation; and starvation, which depletes the carbohydrate reserves of the whole body.

It was therefore considered of interest to discover the effect of ischæmic shock on mitosis, since there are indications that sugar metabolism is profoundly affected in this condition ; moreover, it is also known that there is a gradual, and ultimately a profound, fall in the oxygen consumption of the body at such a time ${ }^{2}$.

A series of experiments has now shown that during the initial stages of ischæmic shock the rate of mitosis is deeply depressed. A typical experiment demonstrating this is summarized in the accompanying table in which two groups, each of five adult male $C B A$ mice, are compared. The first group was an untreated control; but in the second, shock was induced by the application to both hind legs of tourniquets (six turns of a rubber band of size 29), which were removed two hours later at $12.00 \mathrm{hr}$. The rate of mitosis was measured in the ear epidermis by the removal of pieces of ear at intervals from $10.00 \mathrm{hr}$.

The figures show that mitotic activity in the experimental mice fell rapidly to zero after the removal of the tourniquets at $12.00 \mathrm{hr}$. In the control mice, on the other hand, the mitosis-rate rose to a maximum at about $13.00 \mathrm{hr}$., which is a phenomenon associated with the early afternoon sleep period ${ }^{3}$. In normal
Chick test A.O.A.C. $1,500 \pm 100$ $1,400 \pm 100$ 